\title{
Mental aptitude and mnemonic enhancement
}

\author{
DOUGLAS GRIFFITH and TOMME R. ACTKINSON \\ Army Research Institute-Field Unit, Fort Hood, Texas 76544
}

\begin{abstract}
Seventy-two subjects representing equally the general-technical (GT) aptitude ranges 90 and below, 91 to 109 , and 110 and above learned three lists of digit-noun associations. Half the subjects in each group were instructed in a rhyme pegword mnemonic, whereas the remaining subjects served as noninstructed controls. The three lists varied in imagery level, one having high values, one intermediate, and one low. Main effects were obtained for GT level and imagery level, but only the high-GT group (GT $\geqslant 110$ ) was able to employ the mnemonic technique to advantage. This finding questions the generality of some of the imagery literature.
\end{abstract}

The issue of generality is critical to the development of theory in experimental psychology. Unfortunately, the majority of research reported in the experimental journals has employed college students as subjects. Extrapolating from the college population to the general population is not without its risks. In the area of cognition and memory, one of the most potent constructs is imagery. Two common imagery manipulations are instructional set, in which the experimental group is instructed in the use of imagery mnemonic, and item imagery, in which the normative ratings of the imagery. evoking capacity of different items are employed (cf. Paivio, Yuille, \& Madigan, 1968). Typically, subjects instructed in an imagery mnemonic evidence superior recall when compared to a noninstructed control group, and materials rated high on the imagery dimension are recalled much more readily than materials rated low on the dimension. The current experiment seeks to investigate the generality of these findings for military personnel of varying levels of cognitive ability.

Presumably, the Army's General-Technical (GT) test is a measure of general academic ability. The test has an empirical mean of 100 and a standard deviation of 20 . Enlisted personnel were selected from the following three ranges of GT ability: 90 and below, 91 to 109 , and 110 and above. The 110-and-above group is thought to approximate most closely the college population with respect to general academic aptitude. Two instructional conditions, an imagery mnemonic vs. a noninstructed control, and three levels of item imagery were employed with the three GT groups.

\section{METHOD}

\section{Design and Subjects}

A 2 (instructional set) by 3 (GT level) by 3 (imagery level) factorial design was employed. The experimental group was

An earlier version of this paper was presented at the 85th Annual Convention of the American Psychological Association in San Francisco, California, during August 1977. The opinions expressed in this paper are those of the authors and in no way are to be considered as endorsed by the U.S. Army. Requests for reprints should be sent to Douglas Griffith, ARI Field Unit, HQ TCATA (PERI-OH), Fort Hood, Texas 76544. instructed in the use of a rhyme pegword mnemonic, whereas the second group served as a noninstructed control. The three GT levels encompassed the following ranges: 90 and below, 91 to 109 , and 110 and above. Imagery level was manipulated within subjects. Each subject learned three word lists, one each with high, intermediate, and low imagery ratings, respectively. The order of the word lists was fully counterbalanced across subjects in each GT level.

A total of 72 subjects, 24 from each of the GT levels, was employed in the data analysis. Subjects were randomly assigned to conditions with the restriction that an equal number of subjects from each GT level be employed in all conditions. Two subjects, both in the low-GT imagery condition, had to be replaced. One subject did not speak English well enough to serve in the experiment, and the second subject was unable to understand the experimental instructions.

\section{Procedure}

Subjects were run individually. At the outset of the session, the subject was informed that he was participating in a study on memory. Experimental subjects were instructed in the use of a rhyme pegword mnemonic. In this technique, rhyme words are associated with the digits 1 to 10 (e.g., 1 is a bun, 2 is a shoe, etc.). Interacting images are then formed between the rhyme pegwords and the to-be-remembered items.

Initially, the imagery subjects were read the digits and their pegwords. The experimenter then prompted the subject with the digit and asked him to supply the pegword. This procedure was essentially subject paced and each subject was taken to a criterion of two successive perfect recitations. Imagery subjects were then instructed in the technique of forming images between the rhyme pegwords and the to-be-remembered material. First, the technique was illustrated with two response terms. Then each subject was asked to describe the images he formed for three additional high-imagery items. Subjects were provided feedback on their images and the technique was reiterated if it was apparent that the subject did not understand the technique. Subjects were then instructed as to how to employ the technique with abstract words. Subjects were provided with two examples of imagery mediation with abstract words and then were required to describe three additional examples on their own. Feedback was provided after each example.

Control subjects also learned the rhyme pegwords to a criterion of two successive correct repetitions. The cue words for the control subjects, however, were words that rhymed with the pegwords rather than digits (e.g., pun-bun, clue-shoe, etc.). This procedure was followed to keep warm-up and practice effects comparable between the two groups and at the same time to avoid A-B, A-C interference effects that could occur if number-word practice lists were used in the control group. The same response words were used in the sample list for control 
subjects as were used for the imagery subjects during the imagery training.

After the instructional phase, each subject learned three lists, one high imagery, one intermediate imagery, and one low imagery, of 10 words each. The digit-noun pairs were presented at an 8-sec rate in a random order. After all 10 pairs had been presented, a blank slide occurred and the digits were presented individually for $8 \mathrm{sec}$ each. Subjects responded orally. If the subject did not recall at least nine pairs correctly, another blank slide appeared and the study-test cycle was repeated, using a different random order. Altogether, three random orders were employed throughout the experiment. A maximum of five trials was allowed to reach criterion on each list. Approximately $45 \mathrm{sec}$ elapsed between the lists-just enough time to change slide trays.

\section{Lists}

Three lists of items were constructed from the Paivio et al. (1968) norms. Thirty nouns were randomly selected with the following restrictions: (1) that they all have A or AA ThorndikeLorge frequency counts, and (2) that 10 have imagery values less than $3.02,10$ have imagery values between 3.02 and 6.24 , and 10 have imagery values greater than 6.24 . These subsets of 10 items comprised the response terms for the low-, intermediate-, and high-imagery levels, respectively. The words within each subset were then randomly paired with the digits 1-10 with the restriction that there be one noun paired with each digit.

\section{RESULTS}

Two dependent measures were employed, the number of trials to criterion and the number correct on the first trial. However, since both analyses exhibited essentially the same pattern of results, only the latter will be reported. The data are depicted in Figure 1. The effect of item imagery is quite clear from the graph. It is also clear that the instructional manipulation was effective only for the high-GT subjects. A 2 (instructional set) by 3 (GT level) by 3 (imagery level) analysis of variance with repeated measures on the last factor corroborates these impressions. An alpha level of .05 is employed for all statistical tests that follow. The effect of GT level is significant $[F(2,66)=11.46$, MSe $=7.29$ ]. A Newman-Keuls test indicates that the two extreme groups are significantly different, with more words being recalled in the high- than in the lowGT group. The effect of imagery level is also significant $[F(2,132)=36.61, \mathrm{MSe}=2.517]$. The Newman-Keuls test indicates that all three levels differ significantly from each other and that recall is a positive function of the imagery level of the nouns. The effect of instructional set is statistically significant $[F(1,66)=5.03$, $\mathrm{MSe}=7.29]$. This main effect is qualified, however, by the Instructional Set by GT interaction $[F(2,66)=6.94$, $\mathrm{MSe}=7.29$ ]. A test of simple main effects indicates that the mnemonic instruction is effective only for the high-GT group. The remaining $\mathrm{F}$ ratios are less than 1.68 .

\section{DISCUSSION}

This experiment questions the generality of some of the imagery literature. Although the normative manipulation of item imagery proved effective for all GT levels, the instructional

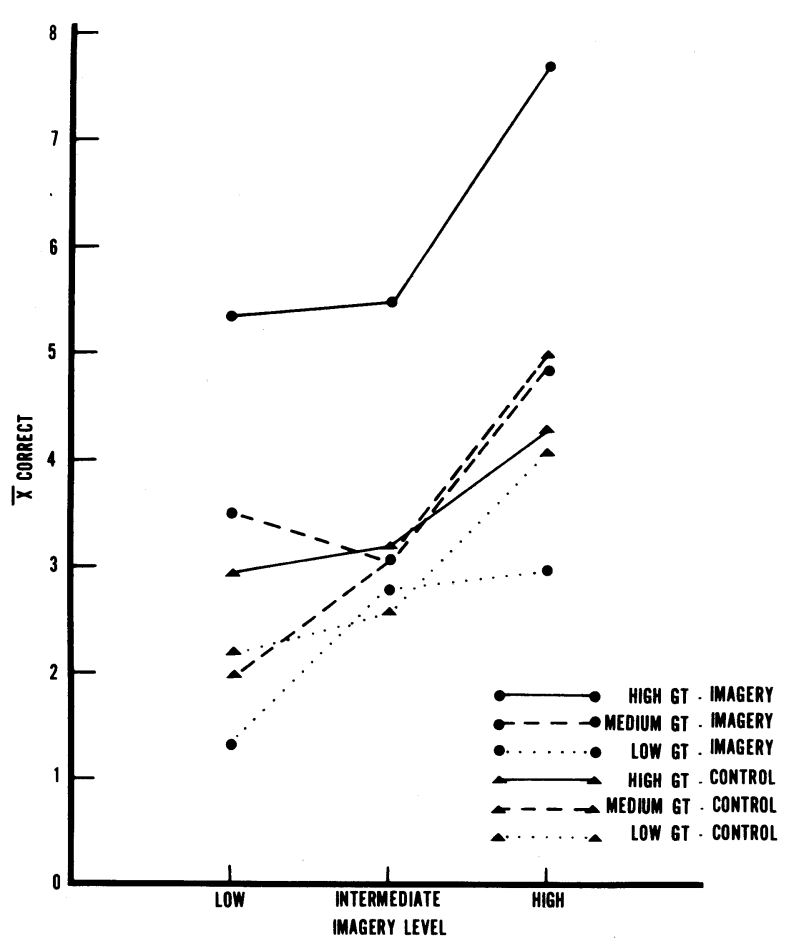

Figure 1. Mean number correct on the first trial.

manipulation did not. Only high-GT personnel, the personnel most resembling the college population with respect to general academic ability, were able to employ the mnemonic technique to advantage. It should be realized that these data should not be employed to conclude that people of low and moderate ability cannot employ mnemonic techniques successfully. Rather, they indicate that in a situation in which college students typically are able to employ a mnemonic technique to advantage (cf. Keppel \& Zavortink, 1969), the general population may not be able to do so.

Another finding of interest is the absence of a significant Instructional Set by Imagery Level interaction. This finding is not unique in that the majority of researchers (cf. Paivio, 1971) have found the effects of instructional set and imagery level to be additive. These two findings, the interaction of instructional set with GT level and the independence of the instructional and the normative manipulations, taken together, indicate that different operational manipulations of the imagery construct are not comparable. That is, the instructional manipulation and the normative manipulation of imagery are by no means equivalent in their effects. Moreover, it is quite likely that the instructional and normative manipulations affect different underlying cognitive processes (cf. Griffith \& Johnston, 1973).

\section{REFERENCES}

Griffith, D., \& Johnston, W. A. An information processing analysis of visual imagery. Journal of Experimental Psychology, 1973, 100, 171-176.

Keppel, G., \& Zavortink, B. Further test of the use of images as mediators. Journal of Experimental Psychology, 1969, 82, 190-192.

Paivio, A. Imagery and verbal processes. New York: Holt, Rinehart, \& Winston, 1971.

Paivio, A., Yuille, J. C., \& Madigan, S. Concreteness, imagery, and meaningfulness values for 925 nouns. Journal of Experimental Psychology Monograph Supplement, 1968, 76(1, Pt. 2).

(Received for publication July $15,1978$. ) 